\title{
Análise do Método Switched Broadcast para Dimensionamento da Rede de um Sistema IPTV
}

\author{
Gisele C. Carmo, Lisandro Lovisolo e João Dias
}

\begin{abstract}
Resumo-O presente trabalho tem como objetivo analisar o método Switched Broadcast e a sua utilização para dimensionamento de canais IPTV (Internet Protocol Television). A principal contribuição deste trabalho é a demonstração, a partir de uma lista de popularidade dos canais de TV por assinatura no Brasil (divulgada pelo IBOPE Instituto Brasileiro de Opinião Pública e Estatística), da viabilidade e do ganho deste método.

Palavras-Chave-IPTV, Switched Broadcast, dimensionamento de canais.

Abstract-The aim of this present work is analyze to Switched Broadcast method and its use for design of IPTV (Internet Protocol Television) channels. The main contribution of this work is the demonstration, from a list of popular channels of pay TV in Brazil (published by IBOPE Brazilian Institute of Public Opinion and Statistics), the feasibility and this method gain.
\end{abstract}

Keywords-IPTV, Switched Broadcast, design of channels.

\section{INTRODUÇÃO}

Impulsionado pela crescente demanda de mídia interativa, a tecnologia IPTV vem despertando muito interesse na comunidade científica [1], principalmente no tocante a largura de banda mínima necessária para atender aos requisitos de QoS (Qualidade de Serviço).

Este trabalho tem por objetivo analisar o método Switched Broadscast [2] para otimização da largura de banda necessária ao serviço IPTV. Estando, para isso, organizado em 5 seções: A seção II apresenta a estrutura de um sistema Switched Broadcast para IPTV e na seção III é descrito a sua modelagem estatística. Na seção IV é abordada a contribuição do Switched Broadcast para o dimensionamento de canais, considerando os canais de TV por assinatura no Brasil, e por fim, na seção V, são apresentadas as conclusões.

\section{Estrutura DE UM SISTEMA SWITCHED BROAdCAST PARA IPTV}

A distribuição de conteúdo de IPTV pode ocorrer através de streaming ao vivo ou sob demanda, como mostra a figura 1.

A recepção pode se dar através de PC (Personal Computer) ou um STB (Set Top Box) com um Televisor.

O Switched Broadcast é um método desenvolvido para a entrega de programação digital, no qual somente é entregue a programação requerida pelo assinante, no momento e lugar em que ele a está requisitando. Os assinantes ficam divididos em grupos denominados nós.

\section{Modelagem eStatístiCA Do Sistema SWITCHED BROADCAST}

A partir da distribuição Zipf [3], que pode ser matematicamente escrita por:

$$
P_{k}=C k^{-\alpha}
$$

Gisele C. Carmo e Lisandro Lovisolo, Departamento de Telecomunicações, Universidade Estadual do Rio de Janeiro. João Dias, Departamento de Telecomunicações, Centro Federal de Educação Tecnológica - Celso Sucow da Fonseca, Rio de Janeiro, Brasil, E-mails: gisele_carmo@yahoo.com.br, lisandro@uerj.br, joaotdias@yahoo.com.br

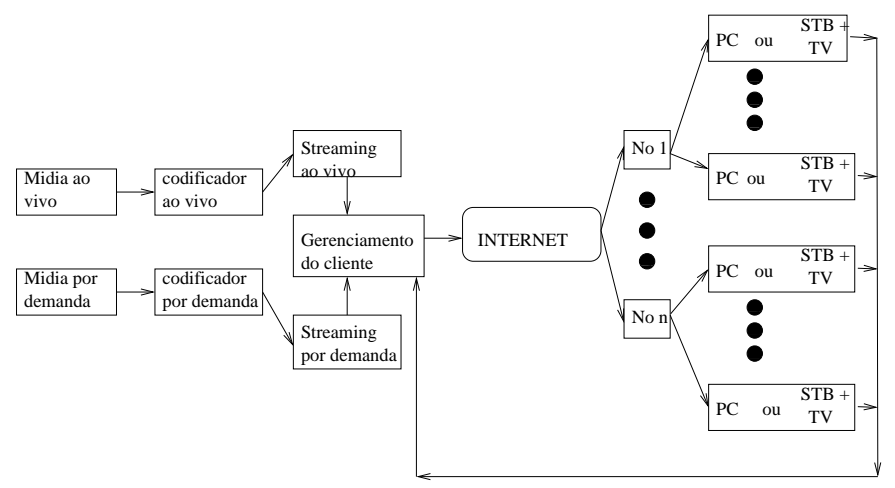

Fig. 1. Distribuição de conteúdo de IPTV pelo método Switched Broadcast.

onde $P_{k}$ representa a probabilidade de alguém estar assistindo o k-ésimo programa de uma lista ordenada pela popularidade. Desta forma, o índice de popularidade de cada emissora de um rank pode ser associado a equação:

$$
N_{k}=A k^{-\alpha}
$$

Reescrevendo a equação (2) de forma linear nos permite calcular a constante $A$ e o termo exponencial $\alpha$, através de regressão linear ou mínimos quadrados.

$$
\log \left(N_{k}\right)=\log (A)-\alpha \log (k)
$$

\section{ApliCAÇÃo Do SWITCHEd BROAdCAST À TV POR ASSINATURA NO BRASIL}

Com o intuito de exemplificar a aplicação do método Switched Broadcast, foi tomada uma situação real à partir da audiência das emissoras que tem sua programação vinculada ao sistema de TV por assinatura no Brasil (veja tabela I, onde foi incluida uma coluna com a probabilidade à partir da distribuição Zipf). Esses dados foram coletados pelo IBOPE em dezembro de 2007 [4].

Aplicando mínimos quadrados, tem-se:

$$
\left\{\begin{array}{l}
\alpha=1,048 \\
A=2,67
\end{array}\right.
$$

Assim,

$$
N_{k}=2,67 k^{-1,048}
$$

O que mostra, na figura (2), ser uma aproximação razoável da distribuição de popularidade da programação.

Calculando a constante $\mathrm{C}$ presente na equação (1), temos:

$$
C=\frac{1}{\sum_{1}^{K} k^{-\alpha}}=0,2388
$$

onde $\mathrm{K}=52$ canais. Desta forma,

$$
P_{k}=0,2388 k^{-1,04819}
$$


TABELA I

RANK DE POPULARIDADE DOS CANAIS DE TV POR ASSINATURA NO BRASIL

\begin{tabular}{|c|c|c|c|}
\hline $\begin{array}{c}\text { rank } \\
(k)\end{array}$ & canais & $\begin{array}{c}\text { aud. } \\
\left(n_{k}\right)\end{array}$ & $\begin{array}{c}\text { prob. } \\
\left(P_{k}\right)\end{array}$ \\
\hline 1 & Cartoon Network & 0,78 & 0.2388 \\
\hline 2 & Discovery Kids & 0,64 & 0.1155 \\
\hline 3 & TNT & 0,55 & 0.0755 \\
\hline 4 & Sport TV & 0,48 & 0.0558 \\
\hline 5 & Globo News & 0,42 & 0.0442 \\
\hline 6 & Nickelodeon & 0,39 & 0.0365 \\
\hline 7 & Telecine Pipoca & 0,37 & 0.0311 \\
\hline 8 & Universal Channel & 0,35 & 0.0270 \\
\hline 9 & Disney Channel & 0,34 & 0.0239 \\
\hline 10 & Multishow & 0,30 & 0.0214 \\
\hline 11 & Telecine Premium & 0,30 & 0.0193 \\
\hline 12 & FOX & 0,29 & 0.0177 \\
\hline 13 & Warner Channel & 0,28 & 0.0162 \\
\hline 14 & Discovery Channel & 0,28 & 0.0150 \\
\hline 15 & AXN & 0,26 & 0.0140 \\
\hline 16 & Telecine action & 0,22 & 0.0131 \\
\hline 17 & Jetix & 0,22 & 0.0123 \\
\hline 18 & Boomerang & 0,21 & 0.0115 \\
\hline 19 & ESPN Brasil & 0,21 & 0.0109 \\
\hline 20 & $\mathrm{HBO}$ & 0,18 & 0.0103 \\
\hline 21 & GNT & 0,18 & 0.0098 \\
\hline 22 & Sport TV 2 & 0,17 & 0.0094 \\
\hline 23 & Sony Entertainment Television & 0,17 & 0.0089 \\
\hline 24 & People + Arts & 0,15 & 0.0085 \\
\hline 25 & National Geographic Channel & 0,13 & 0.0082 \\
\hline 26 & NHK & 0,13 & 0.0079 \\
\hline 27 & Telecine Light & 0,12 & 0.0075 \\
\hline 28 & Animal Planet & 0,11 & 0.0073 \\
\hline 29 & Premiere Futebol Clube (PPV) & 0,10 & 0.0070 \\
\hline 30 & TV Senado & 0,09 & 0.0068 \\
\hline 31 & ESPN & 0,09 & 0.0065 \\
\hline 32 & Telecine Cult & 0,09 & 0.0063 \\
\hline 33 & The History Channel & 0,08 & 0.0061 \\
\hline 34 & Futura & 0,08 & 0.0059 \\
\hline 35 & Max Prime & 0,08 & 0.0057 \\
\hline 36 & $\mathrm{~A}$ and $\mathrm{E}$ & 0,07 & 0.0056 \\
\hline 37 & Cinemax Este & 0,07 & 0.0054 \\
\hline 38 & HBO Plus & 0,07 & 0.0053 \\
\hline 39 & Discovery Home and Healt & 0,06 & 0.0051 \\
\hline 40 & Canal Brasil & 0,06 & 0.0050 \\
\hline 41 & Canal Universitário & 0,06 & 0.0049 \\
\hline 42 & Band News & 0,04 & 0.0047 \\
\hline 43 & El Entertainment Television & 0,04 & 0.0046 \\
\hline 44 & FX & 0,04 & 0.0045 \\
\hline 45 & Shop Time & 0,04 & 0.0044 \\
\hline 46 & Canal Comunitário & 0,03 & 0.0043 \\
\hline 47 & Canal Rural & 0,02 & 0.0042 \\
\hline 48 & MGM & 0,02 & 0.0041 \\
\hline 49 & Bloomberg & 0,02 & 0.0040 \\
\hline 50 & Vh1 & 0,02 & 0.0040 \\
\hline 51 & Band Sports & 0,01 & 0.0039 \\
\hline 52 & Playboy TV & 0,01 & 0.0038 \\
\hline
\end{tabular}

Assim, considerando uma taxa de X Mbps em um canal entre a empresa provedora de conteúdo e um nó onde os usuários estão conectados, pode-se estimar o número máximo de assinantes que essa empresa pode atender neste nó (veja tabela II, onde foi considerado uma taxa de $100 \mathrm{Mbps}$ ), uma vez que

$$
N U_{k}<\frac{1}{P_{k+1}}
$$

onde $N U_{k}$ é o número de usuários assistindo $k$ programas.

Em uma situação inversa, ou seja, sabendo o número de assinantes que se pretende atender, pode-se estimar a largura de banda necessária (veja tabela III, onde foi considerado 200 assinantes por nó), uma vez que

$$
B_{k}=t . k
$$

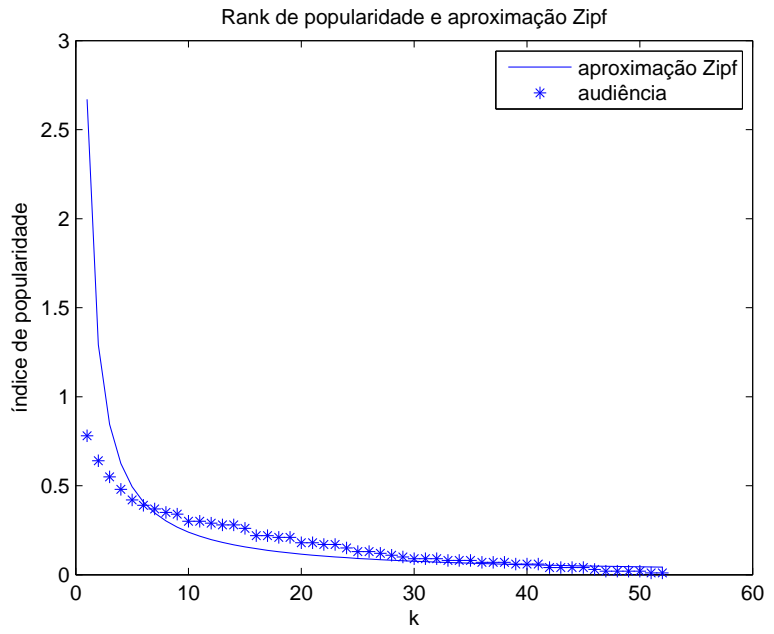

Fig. 2. Rank divulgado pelo IBOPE e aproximação da curva de distribuição Zipf

TABELA II

NÚMERO TOTAL DE ASSINANTES ANTENDIDOS

\begin{tabular}{|c|c|c|c|}
\hline $\begin{array}{c}\text { Tipo de } \\
\text { Compressão }\end{array}$ & $\begin{array}{c}\text { Taxa Média } \\
\text { por Programa }\end{array}$ & $\begin{array}{c}\text { Máximo de } \\
\text { Programas }\end{array}$ & $\begin{array}{c}\text { Usuários } \\
\text { Atendidos }\end{array}$ \\
\hline \hline MPEG 2 & 4 Mbps & 25 canais & 126 \\
\hline H.264 & 2 Mbps & 50 canais & 256 \\
\hline
\end{tabular}

sendo $t$ a taxa média por programa, $k=\left(\frac{C}{P_{k}}\right)^{\frac{1}{\alpha}}$ e $P_{k}$ obtido pela equação 8 .

TABELA III

\begin{tabular}{|c|c|c|c|}
\hline $\begin{array}{c}\text { Tipo de } \\
\text { Compressão }\end{array}$ & $\begin{array}{l}\text { Taxa Média } \\
\text { por Programa }\end{array}$ & $\begin{array}{l}\text { Máximo de } \\
\text { Programas }\end{array}$ & $\begin{array}{l}\text { Largura } \\
\text { de banda }\end{array}$ \\
\hline MPEG 2 & 4 Mbps & 40 canais & $160 \mathrm{Mbps}$ \\
\hline H.264 & $2 \mathrm{Mbps}$ & 40 canais & $80 \mathrm{Mbps}$ \\
\hline
\end{tabular}

LARGURA DE BANDA NECESSÁRIA

\section{CONCLUSÕES}

O método Switched Broadcast foi analisado como uma ferramenta que possibilita o dimensionamento do canal para entrega de programas em sistemas IPTV. Através de uma análise do rank de popularidade divulgado pelo IBOPE, pôdese ter uma idéia da banda necessária para a transmissão dos programas, de quantos usuários poderiam ser atendidos e viceversa.

\section{REFERÊNCIAS}

[1] Albuquerque, C., Proença, T., Oliveira, E., TVoIP: TV sobre IP: Arquiteturas para Transmissão em Larga Escala. Minicurso, Simpósio Brasileiro de Redes de Computadores (SBRC), 2006.

[2] Vasudevan, S. V., Switched Broadcast: Statistics From the Field, Big Band Networks, 13 mai. 2003.

[3] Powers, D. M. W., Applications and Explanations of Zipfś Law, NeMLaP3/CoNLL98: New Methods in Language Processing and Computational Natural Language Learning, ACL, pp 151-160, 1988.

[4] Audiência - Os 52 canais mais assistidos dentro e fora do Brasil, IBOPE, 20 jan. 2008. 